\title{
Transient expression of calretinin in the trout habenulo-interpeduncular system during development
}

Authors:

Angel Porteros, Jesús G. Briñón, R. Arévalo, C. Crespo, J. Aijón and José R. Alonso

Running title:

CR in the habenulo-interpeduncular system

Institutional affiliation:

Departamento de Biología Celular y Patología.

Universidad de Salamanca.

E-37007 Salamanca. Spain.

Correspondence to be sent to:

Dr. José R. Alonso.

Departamento de Biología Celular y Patología.

Universidad de Salamanca.

Facultad de Medicina.

C/ Alfonso X el Sabio, 1.

E-37007 Salamanca. Spain.

Phone: +34-23-294400 ext. 1854

Fax: +34-23-294549

E-mail: jralonso@gugu.usal.es 


\begin{abstract}
Calcium-binding proteins control calcium homeostasis during neural development. The expression of one of these proteins, calretinin (CR), was monitored by immunohistochemistry in the developing habenulo-interpeduncular system of the rainbow trout, a conserved region of the brain along vertebrate phylogeny that undergoes a neurochemical reorganization in late development. No CR-immunoreactivity was observed in the habenulo-interpeduncular system during the embryonic development. CRimmunolabeling appeared in newly hatched fry, and during the fry development the number of CR-immunostained elements increased progressively. During the juvenile stages (from 30 days post-hatching onwards) a gradual decrease in the number of CRimmunostained cells occurred, until its complete disappearance in adults. These variations in CR expression may represent variable calcium-buffering needs during different developmental stages.
\end{abstract}

\title{
KEY WORDS
}

Anamniote, Calcium, Calcium-binding protein, Development, Fish, Immunohistochemistry 
Calretinin (CR) is an EF-hand calcium-binding protein of $29 \mathrm{KDa}$ molecular weight identified by cloning cDNA of the chick retina [17]. The conserved structure of CR throughout evolution [15] suggests that it should have a key role in calcium homeostasis in different vertebrate species. CR fluctuactions have been reported in the developing brain of amniotes [1] suggesting a relationship between CR expression and the control of intracellular free calcium levels during development.

The habenulo-interpeduncular system is a conserved part of the brain along the phylogeny, that consists of the habenula connected to the interpeduncular nucleus of the mesencephalon by the habenulo-interpeduncular tract, also named fasciculus retroflexus. Input from olfactory and limbic areas of the telencephalon that project to the habenula reaches the mesencephalon by this route, and integrates with other sensory information to coordinate the appropriate motor response [3]. Although the basic cytoarchitecture of this pathway in the brain is constituted at birth, synapse formation and maturation continue post-natally [10].The habenular and interpeduncular nuclei also undergo a neurochemical reorganization during postnatal development in both amniotes [9] and anamniotes [2,6,12]. Although CR is considered a good indicator of the maturation stage of neural systems [5], its distribution has been studied only in the adult habenulo-interpeduncular pathway [18] and there are no available data for the ontogeny of this system in any species.

Fertilized eggs of rainbow trout (Oncorhynchus mykiss)were kept in aquaria with fresh water and maintained at $10^{\circ} \mathrm{C}$ with a $12: 12$ hour light:dark cycle. Developing trout were staged as previously [16]. Three animals from each of the following stages were used: 250 day-degrees (dd), 300 dd and 350 dd embryos; newly hatched ( $\sim 400$ dd), 6 days after hatching (D) and 21 D fry; 30 D, 90 D, 180 D and one year old juveniles; and adults (three years old).

Animals were anesthetized with 0.03\% MS-222 (Sigma). Embryos and fry were fixed by immersion in 4\% paraformaldehyde and 2\% picric acid in $0.1 \mathrm{M}$ phosphate buffer, pH 7.4 (PB) for 4 hours. Juveniles and adults were perfused transcardially with $0.63 \%$ 
$\mathrm{NaCl}$ solution followed by the same fixative. Following cryoprotection with $30 \%$ sucrose (w/v) in PB, frontal sections were cut at $20 \mu \mathrm{m}$ on a Leica cryostat and mounted on slides. For CR immunohistochemistry, tissue was pre-incubated with $10 \%$ normal goat serum and $0.1 \%$ Triton $\mathrm{X}-100$ in $\mathrm{PB}$ for 1 hour at $4^{\circ} \mathrm{C}$. The tissue was then incubated for two days at $4^{\circ} \mathrm{C}$ with rabbit anti-CR serum $(1: 10,000)$ that has been fully characterized [17] and previously used in teleostean brain [16]. After 1 hour in biotinylated goat anti-rabbit immunoglobulin G (Vector Laboratories;1:200), and 2 hours in avidin-biotin-peroxidase complex (Vector Labs.; 1:225), tissue-bound peroxidase was visualized with 0.07\% 3,3'diaminobenzidine (Sigma) and $0.003 \% \mathrm{H}_{2} \mathrm{O}_{2}$. Sections were dehydrated and coverslipped with Entellan (Merck). The anti-CR serum specificity was tested by preadsorption with native CR $(2.5 \mu \mathrm{g} / \mathrm{ml})$, obtaining a complete abolition of the immunohistochemical staining. In addition, three controls of the immunohistochemical procedure were carried out by incubating sections in the same media omitting: 1) primary antibody; 2) biotinylated immunoglobulin, and 3) avidin-biotin-peroxidase complex. No residual reaction was observed. The density of CR-immunoreactive neurons was evaluated estimating the ratio between the number of CR-immunostained cell bodies and the area of each nucleus calculated in $\mu^{2}$ of fixed and immunostained tissue, within 3 randomly selected sections per animal. For this purpose we used a digitizer tablet connected to a image analysis system (MOP-Videoplan Kontron). Maximum diameters of 50 cells per stage were measured using a Zeiss ocular micrometer.

We observed CR-immunostaining in the habenula and interpeduncular nucleus of the rainbow trout at different stages after hatching. No CR-immunoreactivity was observed in any of the embryonic stages analyzed (250 dd, $300 \mathrm{dd}$, and $350 \mathrm{dd}$ ).

CR immunolabeling presented a simultaneous onset in both the habenula and the interpeduncular nucleus, and it was first observed in newly hatched fry ( $\sim 400 \mathrm{dd})$. In the habenula, CR-immunoreactive cells appeared in the dorsal region (Fig. 1a). These cells were small (6-8 $\mu$ m maximum diameter), round and without immunostained prolongations. In the interpeduncular nucleus, CR-immunoreactive cells appeared as a unique mass 
located close to the midline of the caudalmost part of the mesencephalon (Fig. 2a). The immunoreactive cells were of similar shape and size to those observed in the habenula.

During the fry development, the CR-immunoreactive cells shared the morphological features described for the newly hatched animals in both the habenula and the interpeduncular nucleus. There was, however, a gradual increase in the size and number of the CR-immunostained neurons (Table 1). The increase in number had already been observed in $6 \mathrm{D}$ fry (Figs. 1b, 2b). At this stage, the dorsal and ventral parts of the interpeduncular nucleus could be differentiated, with CR-immunoreactive cells (7-9 $\mu \mathrm{m}$ maximum diameter) scattered in the whole extension of both parts of the nucleus (Fig. 2b). At the $21 \mathrm{D}$ stage, CR-immunostained cells were slightly bigger (8-10 $\mu \mathrm{m}$ maximum diameter) and more abundant than in the $6 \mathrm{D}$ stage in both the habenula (Fig. 1c) and the interpeduncular nucleus (Fig. 2c), and their CR-immunostaining intensity was also stronger. In addition, CR-immunolabeling could be observed in the neuropilar area of the interpeduncular nucleus, with more intensity in the ventral than in the dorsal half (Fig. 2c).

During the juvenile stages, the shape and size of CR-immunostained cells remained almost constant (around $10 \mu \mathrm{m}$ maximum diameter), and a gradual decrease in the number of labelled cells occurred in both the habenula and the interpeduncular nucleus (Table 1). At the $30 \mathrm{D}$ stage, we observed a lower number of CR-immunopositive elements than in the previous stage studied (21 D). The decrease was more evident in the $90 \mathrm{D}$ juveniles (Figs. 1d, 2d). In addition, CR-immunoreactive cells had an uneven distribution in the interpeduncular nucleus. CR-immunoreactive neurons were located at the external limits of the nucleus, especially in its dorsal half (Fig. 2d). In the one year old juveniles, the number of CR-immunoreactive cells was very low in both the habenula and the interpeduncular nucleus. In the former, CR-immunoreactive cells were arranged in clusters in the lateral part (Fig. 1e). In the interpeduncular nucleus, the positive cells were located in the outermost region in both dorsal and ventral parts, whereas the regions close to the mesencephalic midline were practically devoid of CR-immunolabeled cells (Fig. 2e). In the adult, CR-immunoreactivity was completely absent in the habenula (Fig. 1f) and the 
interpeduncular nucleus (Fig. 2f). Apart from the disappearance of CR-immunolabeled neurons, the labeling of the neuropilar area of the interpeduncular nucleus was progressively reduced until its disappearance in the adult (Fig. 2f), following a temporal pattern similar to that observed in the cell labeling.

The neurochemistry of the habenulo-interpeduncular system has been extensively studied in mammals, but it is still poorly analyzed in non-mammalian vertebrates. In teleosts, immunohistochemical studies have indicated the presence of substance P [19], choline acetyltransferase and nitric oxide synthase [20], serotonin [6], neuropeptide Y [4], and bombesin [8]. With respect to CR, there are no available data on their distribution during the habenulo-interpeduncular system development of any species. In this study we report that CR does not appear in the embryo or in the adult rainbow trout, but it can be immunohistochemically detected just after hatching. The CR expression develops in a progressive manner during the fry development, and it decreases gradually within a long period of juvenile development until its complete loss at adulthood. Transient expression of CR has also been observed in other regions of the amniote brain [1].

Different mechanisms may account for such transient expression. The disappearance of CR may be due to cell death, to changes in neurochemical phenotype or to cell migration to a different location in the adult animal. With respect to a loss of CRimmunopositive cells, we did not observe immunoreactive cells with degenerate or shrunken somata that could identify cells dying during brain development [14]. Nevertheless, we cannot exclude that the loss of CR may occur immediately before the onset of degeneration. Therefore, neuronal death could be an active process in which new gene expression is initiated and triggers a cascade of specific events that actively induce degeneration and death [13]. One of these events could be the termination of CR mRNA transcription, with a consequent loss of calcium-buffering, resulting in an increase in the intracellular concentration of free calcium, causing cell death. With respect to neuronal migration, interpeduncular CR-immunoreactive neurons were progressively observed in the periphery of the nucleus, but even these displaced neurons stop the expression of CR in 
the adult stage, since they were no longer observed in the studied nuclei or in adjacent areas.

The most likely explanation for this phenomenon is that there is a down-regulation of CR expression after $30 \mathrm{D}$. Neuronal differentiation not only entails the acquisition of various molecular and cellular characteristics but it also involves the loss of neurochemical characteristics that appear only during certain stages of development [14]. Although the physiological significance of the transient expression of calcium-binding proteins remains unknown, there are evidences suggesting that their expression may be modulated by stimuli causing a variation in the concentration of cytosolic calcium [11], indicating that changes in neuronal activity may regulate the expression of these proteins [7]. In this sense, the habenulo-interpeduncular system of salmonid teleosts undergoes drastic neural changes during the "midlife neuroembryonic period", in which olfactory imprinting takes place and behaviour changes [6]. These changes lead to a transient expression of neuroactive substances such as tyrosine hydroxylase [12] or serotonin [6] in the habenula and somatostatin in the interpeduncular nucleus [2] and CR in both the habenula and the interpeduncular nucleus (present report). During this period, neurons in the habenulointerpeduncular system may need a more refined mechanism of calcium buffering (represented by the transient expression of CR) than in the adult.

The authors thank Dr. J. Rogers for kindly providing the anti-CR serum. Mr. G. H. Jenkins revised the English version of the manuscript. Supported by the DGES (PB971341) and the Junta de Castilla y León. 
[1] Bastianelli, E. and Pochet, R., Transient expression of calretinin during development of chick cerebellum. Comparison with calbindin-D28k, Neurosci. Res., 17 (1993) 53-61.

[2] Becerra, M., Manso, M.J., Rodríguez-Moldes, I. and Anadón, R., Ontogeny of somatostatin-immunoreactive systems in the brain of the brown trout (Teleostei), Anat. Embryol., 191 (1995) 119-137.

[3] Butler, A.B. and Hodos, W., Comparative Vertebrate Neuroanatomy: Evolution and Adaptation, Wiley-Liss, 1996, 213-214 pp.

[4] Chiba, A., Sohn, Y.C. and Honma, Y., Distribution of neuropeptide Y and gonadotropin-releasing hormone immunoreactivities in the brain and hypophysis of the ayu, Plecoglossus altivelis (Teleostei), Arch. Histol. Cytol., 59 (1996) 137-148.

[5] Dechesne, C.J., Rabejac, D. and Desmadryl, G., Development of calretinin immunoreactivity in the mouse inner ear, J. Comp. Neurol., 346 (1994) 517-529.

[6] Ebbesson, L.O.E., Holmqvist, B., Östholm, T. and Ekström, P., Transient serotoninimmunoreactive neurons coincide with a critical period of neural development in coho salmon (Oncorhynchus kisutch), Cell Tissue Res., 268 (1992) 389-392.

[7] Friauf, E., Transient appearance of calbindin-D-28k-positive neurons in the superior olivary complex of developing rats, J. Comp. Neurol., 334 (1993) 59-74.

[8] Himick, B.A. and Peter, R.E., Bombesin-like immunoreactivity in the forebrain and pituitary and regulation of anterior pituitary hormone release by bombesin in goldfish, Neuroendocrinology, 61 (1995) 365-376.

[9] Joyce, M.P. and Barr, G.A., The development of cholecystokinin in the interpeduncular nucleus of rats, Dev. Brain. Res, 65 (1992) 275-279.

[10] Lenn, N.J., Postnatal synaptogenesis in the rat interpeduncular nucleus, J. Comp. Neurol., 181 (1978) 75-92.

[11] Lowenstein, D.H., Miles, M.F., Hatam, F. and McCabe, T., Up regulation of calbindin-d28k mRNA in the rat hippocampus following focal stimulation of the perforant path, Neuron, 6 (1991) 627-633. 
[12] Manso, M.J., Becerra, M., Molist, P., Rodríguez-Moldes, I. and Anadón, R., Distribution and development of catecholaminergic neurons in the brain of the brown trout. A tyrosine hydroxylase immunohistochemical study, J. Hirnforsch., 2 (1993) 239-260.

[13] Oppenheim, R.W., Prevette, D., Tytell, M. and Homma, S., Naturally occurring and induced neuronal death in the chick embryo in vivo requires protein and RNA synthesis, Dev. Biol., 138 (1990) 104-113.

[14] Oppenheim, R.W., Cell death during development of the nervous system, Annu. Rev. Neurosci., 14 (1991) 453-501.

[15] Parmentier, M., Structure of the human cDNAs and genes coding for calbindin D28k and calretinin. In R. Pochet, D.E.M. Lawson and C.W. Heizmann (Eds.), Calcium Binding Proteins in Normal and Transformed Cells, Plenum Press, New York, 1990, pp. 27-34.

[16] Porteros, A., Arévalo, R., Weruaga, E., Briñón, J.G., Crespo, C., Alonso, J.R. and Aijón, J., Calretinin immunoreactivity in the developing olfactory system of the rainbow trout, Dev. Brain Res., 110 (1997) 101-109.

[17] Rogers, J.H., Calretinin: a gene for a novel calcium-binding protein expressed principally in neurons, J. Cell Biol., 105 (1987) 1343-1353.

[18] Rogers, J.H. and Résibois, A., Calretinin and calbindin D-28k in rat brain: patterns of partial co-localization, Neuroscience, 51 (1992) 843-865.

[19] Villani, L., Guarnieri, T., Salsi, U. and Bollini, D., Substance P in the habenulointerpeduncular system of the goldfish, Brain Res. Bull., 26 (1991) 225-228.

[20] Villani, L., Guarnieri, T. and Zironi, I., Choline acetyltransferase and NADPHdiaphorase localization in the goldfish habenulo-interpeduncular system, Neurosci. Lett., 173 (1994) 67-70. 


\section{TABLE}

Table I. Density (mean \pm s.e.m.) of CR-immunostained cells in the habenulo-interpeduncular system (cells per $10^{4} \mu^{2}$ )

\begin{tabular}{lcc} 
Stage & Habenula & Interpeduncular nucleus \\
\hline-050 dd & 0 & 0 \\
300 dd & 0 & 0 \\
350 dd & 0 & 0 \\
Newly hatched (400 dd) & $8.6 \pm 0.9$ & $16.1 \pm 0.8$ \\
6 D & $13.5 \pm 0.7$ & $37.5 \pm 1.2$ \\
21 D & $18.7 \pm 1.1$ & $58.1 \pm 1.4$ \\
30 D & $15.3 \pm 0.8$ & $50.2 \pm 0.9$ \\
90 D & $12.8 \pm 0.6$ & $42.3 \pm 0.8$ \\
180 D & $10.7 \pm 0.9$ & $34.7 \pm 1.3$ \\
One year & $5.4 \pm 0.6$ & $14.9 \pm 0.6$ \\
Three years (adult) & 0 & 0
\end{tabular}




\section{FIGURE LEGENDS}

\section{Figure 1}

Transient expression of CR in the habenula of the rainbow trout. First CRimmunoreactive elements appeared at the hatching stage (a). The number of CRimmunolabeled elements increased progressively in the $6 \mathrm{D}$ (b) and $21 \mathrm{D}$ (c) stages, but progressively decreased during the juvenile development, as observed in $90 \mathrm{D}$ (d) and one year old (e) juveniles, until the complete abolition in adults (f). Scale bar $=50 \mu \mathrm{m}$ for all figures.

\section{Figure 2}

Transient expression of CR in the interpeduncular nucleus of the rainbow trout. CR-immunolabeling was first detected in newly hatched fry (a) and it increased progressively in $6 \mathrm{D}$ (b) and $21 \mathrm{D}$ (c) stages. The number of cells decreased gradually during the juvenile development, as observed in $90 \mathrm{D}(\mathrm{d})$ and one year old (e) specimens, and disappeared completely in adults (f). Scale bar in a,b $=50 \mu \mathrm{m}$; in c-f $=100 \mu \mathrm{m}$. 
$-\ldots-a=b \cdot$ $-\sin 2$
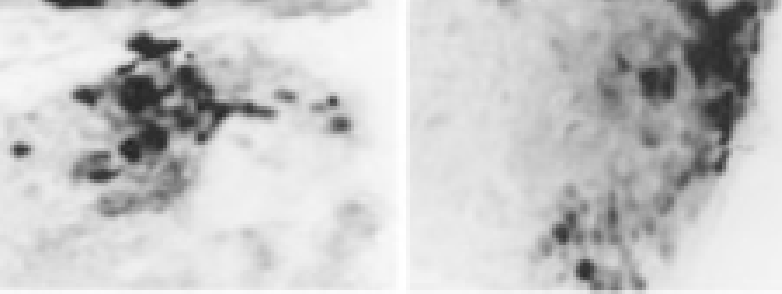

c

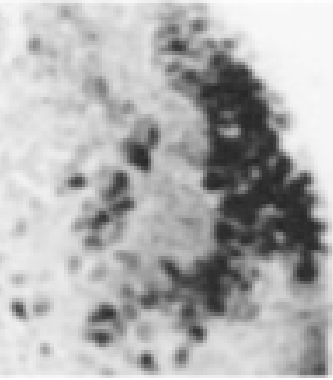

d

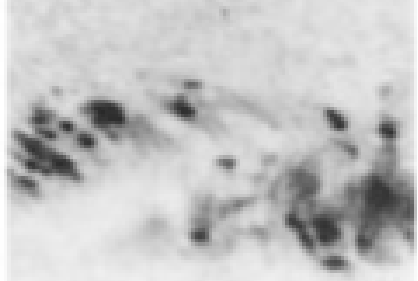

e
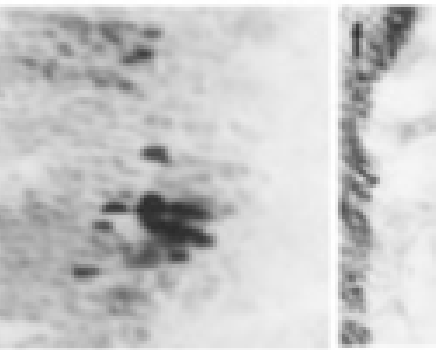
a

o

$2 x^{2}$

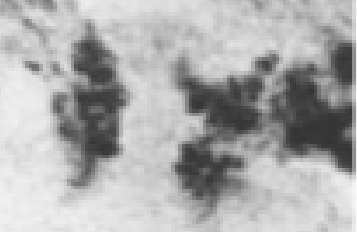

$\sqrt{2}+x^{2}$

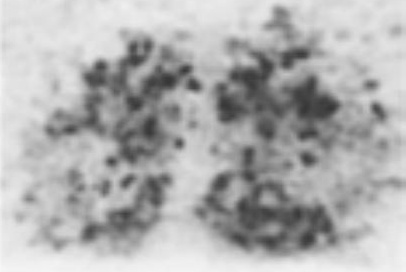

\section{citafr:}

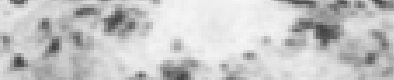

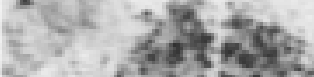

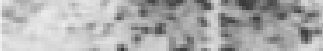

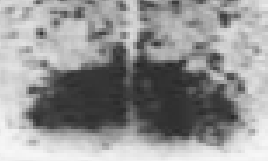

i

$\because$

$+8$

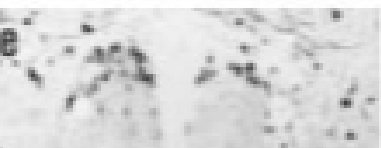

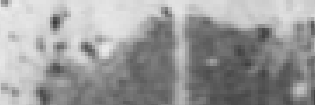

$\infty$

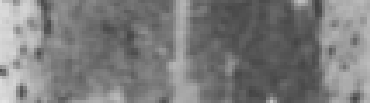

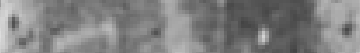
it

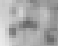

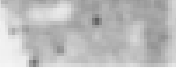

$\mathrm{RP}$ 